\title{
Genetically determined variation in the number of phenotypically defined hematopoietic progenitor and stem cells and in their response to early-acting cytokines
}

\author{
Els Henckaerts, Hartmut Geiger, J essica C. Langer, Patricia Rebollo, Gary Van Zant, and Hans-Willem Snoeck
}

\begin{abstract}
Quantitative trait analysis may shed light on mechanisms regulating hematopoiesis in vivo. Strain-dependent variation existed among C57BL/6 (B6), DBA/2, and $B X D$ recombinant inbred mice in the responsiveness of primitive progenitor cells to the early-acting cytokines kit ligand, flt3 ligand, and thrombopoietin. A significant quantitative trait locus was found on chromosome 2 that could not be confirmed in congenic mice, however, probably because of epistasis. Because it has been shown that alleles of unknown Xlinked genes confer a selective advantage to hematopoietic stem cells in vivo in humans and in cats, we also analyzed
\end{abstract}

reciprocal male D2B6F1 and B6D2F1 mice, revealing an $X$-linked locus regulating the responsiveness of progenitor and stem cells to early-acting factors. Among DBA/2, B6, and BXD recombinant inbred mice, correlating genetic variation was found in the absolute number and fre-

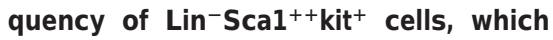
are highly enriched in hematopoietic progenitor and stem cells, and in the number of $\mathrm{Lin}^{-} \mathrm{Scal}^{++}$kit $^{-}$cells, a population whose biologic significance is unknown, suggesting that both populations are functionally related. Suggestive quantitative trait loci (QTLs) for the number of Lin-Sca1 $^{++}$cells on chromosomes 2, 4, and 7 were confirmed in successive rounds of mapping. The locus on chromosome 2 was confirmed in congenic mice. We thus demonstrated genetic variation in the response to cytokines critical for hematopoiesis in vivo and in the pool size of cells belonging to a phenotype used to isolate essentially pure primitive progenitor and stem cells, and we identified loci that may be relevant to the regulation of hematopoiesis in steady state. (Blood. 2002;99:3947-3954)

다 2002 by The American Society of Hematology

\section{Introduction}

Hematopoiesis consists of an ordered series of events in which primitive hematopoietic stem cells renew or differentiate into mature blood cells of at least 8 lineages. The exact mechanisms responsible for the regulation of self-renewal versus differentiation of hematopoietic stem cells in vivo are unknown but may involve stochastic mechanisms and a balance between stimulatory and inhibitory signals regulating renewal, differentiation, and apoptosis. ${ }^{1}$

One strategy to gain insight into the regulation of hematopoiesis is to study naturally occurring genetic variation in the hematopoietic system. This may lead to the identification of regulatory mechanisms that are relevant in vivo. Pool size and cycling activity of the stem cell compartment are under complex intrinsic genetic control in inbred mouse strains. ${ }^{2-8}$ Putative stem cell pool size, as determined by the day 35 cobblestone area-forming cell assay (CAFCd35), varies widely among inbred mouse strains. ${ }^{2}$ This was not the case for earlier appearing, and therefore more mature, CAFCd7, indicating that the gene(s) involved act on the more primitive progenitor compartment. ${ }^{8}$ Using BXD recombinant inbred (RI) mouse strains, a locus involved in the regulation of CAFCd35 frequency was mapped to mouse chromosome 18, in a region syntenic with and corresponding to a critical segment of human chromosome $5 q$, which is frequently deleted in myelodys- plasia and acute myeloblastic leukemia. ${ }^{2}$ Muller-Sieburg and Riblet, using a different assay in BXD mice, mapped 2 candidate loci regulating the number of long-term culture-initiating cells (LTC-IC) to mouse chromosome $1 .^{3}$ Further evidence for the existence of intrinsic genetic control of hematopoietic stem cell kinetics comes from embryo-aggregated chimeric DBA/ $2 \leftrightarrow \mathrm{B} 6$ mice. In these mice, DBA/2-derived stem cells contribute significantly and stably to hematopoiesis in young adults, but with aging, B6-derived hematopoiesis becomes predominant, if not exclusive. ${ }^{5}$ Hematopoietic recovery after the administration of the myelotoxic drug 5-fluorouracil to young $\mathrm{B} 6 \leftrightarrow \mathrm{DBA} / 2$ chimeras was significantly faster in the DBA/2-derived stem cell compartment than in the B6-derived compartment. ${ }^{6}$ Similarly, when chimeric bone marrow was transplanted, early engraftment of irradiated hosts was predominantly DBA/2 derived, significantly out of proportion to the DBA/2 representation in the marrow graft, further suggesting a proliferative advantage for DBA/2-derived stem cells. Because the microenvironment in these chimeric mice was identical for both DBA/2- and B6-derived stem cells, stem cell-intrinsic mechanisms must be at the basis of these differences. ${ }^{6}$ Genetic variation has also been demonstrated in the repopulation capacity of hematopoietic stem cells. One of the CXB RI strains had a significantly higher
From the Carl C. Icahn Institute for Gene Therapy and Molecular Medicine, Mount Sinai School of Medicine, New York, NY; and the Markey Cancer Center, University of Kentucky, Lexington.

Submitted October 18, 2001; accepted J anuary 23, 2002.

Supported in part by National Institutes of Health grant RO1 AG16327 (H.-W.S.) and RO1 AG16653 (G.V.Z.). E.H. was supported by a fellowship from the Belgian American Educational Foundation and by the Belgian Hematological Society. H.G. was supported by a fellowship from the Deutsche
Akademie der Naturforscher Leopoldina.

Reprints: Hans-Willem Snoeck, Carl C. Icahn Institute for Gene Therapy and Molecular Medicine, Mount Sinai School of Medicine, Box 1496, One Gustave L. Levy PI, New York, NY 10029; e-mail: hans.snoeck@mssm.edu.

The publication costs of this article were defrayed in part by page charge payment. Therefore, and solely to indicate this fact, this article is hereby marked "advertisement" in accordance with 18 U.S.C. section 1734.

(c) 2002 by The American Society of Hematology 
capacity for long-term competitive repopulation in CByB6F1 recipient mice than any of the other CXB strains. ${ }^{7}$ Loci affecting the efficiency of mobilization of progenitor cells to the peripheral blood have been identified on chromosomes 2 and $11 .{ }^{9}$

In humans and in cats, evidence for genetically determined regulation of hematopoiesis comes from studies addressing $\mathrm{X}$ inactivation in the hematopoietic system in vivo. ${ }^{10-13}$ In as many as $50 \%$ of aging women, progressive skewing of $\mathrm{X}$ inactivation occurs in the hematopoietic system. . $^{10,11}$ Similar data were obtained in cats. ${ }^{12}$ Furthermore, skewed $\mathrm{X}$ chromosome inactivation in all hematopoietic lineages also occurs after bone marrow transplantation $^{12}$ and after repeated chemotherapy with busulfan in cats. ${ }^{13,14}$ Human female monozygotic twins that show skewed $\mathrm{X}$ inactivation in the hematopoietic system with aging tend to inactivate the $\mathrm{X}$ chromosome from the same parent. ${ }^{11,15}$ Thus, alleles on the $\mathrm{X}$ chromosome confer a growth, survival, or reconstitution advantage to stem cells. Progressive skewing of $\mathrm{X}$ inactivation in the hematopoietic system is therefore likely to be caused to a large extent by hemizygous selection, ${ }^{12}$ ie, a selective advantage of one $\mathrm{X}$ chromosome over another, whereas stochastic mechanisms are less dominant. ${ }^{15}$

The aim of this study was to test the hypothesis that straindependent variation would exist in the response of primitive hematopoietic progenitor and stem cells, as defined by the $\mathrm{Lin}^{-} \mathrm{Sca} \mathrm{1}^{++} \mathrm{kit}^{+}$phenotype, ${ }^{16-18}$ to the early-acting factors kit ligand (KL), flt3 ligand (ftt3L), and thrombopoietin (TPO). These factors have been shown to be critical to the function of the hematopoietic stem cell compartment in vivo. ${ }^{19-21}$ We show here that there is indeed strain-dependent variation in the response to these early-acting factors and that at least one locus for this trait maps to the $\mathrm{X}$ chromosome. In addition, we found strain-dependent variation in the absolute number and frequency of $\mathrm{Lin}^{-} \mathrm{Sca} 1^{++}$ cells (c-kit ${ }^{+}$and c-kit ${ }^{-}$subpopulations). A quantitative trait locus (QTL) for this trait on chromosome 2 was confirmed in congenic mice.

\section{Materials and methods}

\section{Mice}

Female mice of strains C57BL/6J (B6), DBA/2J, and BXD RI, aged 6 to 8 weeks, were all purchased from Jackson Laboratories (Bar Harbor, ME). The mice were maintained in a germ-free environment and fed ad libitum. Experiments and animal care were performed in accordance with the Mount Sinai Institutional Animal Care and Use Committee.

\section{Antibodies and cytokines}

Unconjugated Ter119 (erythroid), CD2 (T and NK cells), CD3, CD4, CD8 (T cells), B220 (B cells), Ly6G/Gr1 (granulocytes), Mac1 (macrophages), phycoerythrin-conjugated Sca1, biotin-conjugated anti-c-kit, Cychromeconjugated streptavidin, and fluorescein isothiocyanate-conjugated goat antirat antibodies were purchased from PharMingen (San Diego, CA) Recombinant mouse flt3L and thrombopoietin (TPO) and anti-transforming growth factor- $\beta$ (anti-TGF- $\beta$ ) antibodies were purchased from R\&D Systems (Minneapolis, MN). Supernatants from BHK/HM-5, BHK/MKL (both a kind gift of Dr J. Matous, University of Washington), and WEHI 3B (a kind gift of Dr S Tsai, Mount Sinai School of Medicine, New York, NY) cells were used as a source of granulocyte macrophage-colony-stimulating factor (GM-CSF), KL, and interleukin-3 (IL-3), respectively.

\section{Isolation of Lin-Sca1 ${ }^{+/+}$cells}

Femurs and tibias were flushed with Iscoves modified Dulbecco medium (IMDM; Gibco BRL, Grand Island, NY) supplemented with 10\% fetal calf serum (FCS). Low-density bone marrow cells, obtained after density centrifugation, were stained with Ter119, CD2, CD3, CD8, CD4, B220, Mac1, and $\mathrm{Gr} 1$ for 20 minutes at $4^{\circ} \mathrm{C}$, washed, and stained with goat antirat antibodies for 20 minutes at $4^{\circ} \mathrm{C}$. After washing, the cells were stained for 20 minutes at $4^{\circ} \mathrm{C}$ with phycoerythrin-conjugated Scal and biotinconjugated CD117 (c-kit), washed with phosphate-buffered saline, and stained with streptavidin-Cychrome. Cells were sorted on a MoFlo flow cytometer (Cytomation, Fort Collins, CO) at 30 psi sheath pressure and at a rate of 12000 to 15000 events per second. Cells with a low side scatter, a low-to-medium forward scatter (Figure 1, R1), a green (lineage) fluorescence lower than the median fluorescence of cells stained with isotypematched control antibodies, and an orange (Sca1) fluorescence twice the intensity (in terms of channel numbers) of the brightest cells in control samples (Figure 1, R2) were sorted as Lin $^{-}$Sca1 ${ }^{++}$cells. Cells falling in R1 and R2 with positive red (c-kit) fluorescence (Figure 1, R3) were sorted as $\mathrm{Lin}^{-} \mathrm{Sca}^{++}{ }^{+} \mathrm{kit}^{+}$cells, whereas cells falling in R1 and R2 with negative red fluorescence (Figure 1, R4) were sorted as $\mathrm{Lin}^{-} \mathrm{Sca}^{++}{ }^{+} \mathrm{kit}^{-}$cells. The absolute number of cells in each population was estimated by recording the number of sorted cells, as indicated by the number of sortable events on the counter of the cell sorter. The abort frequency was less than $5 \%$ in the sorting conditions we used. Bone marrow pooled from at least 2 mice was used in each experiment. Because the secondary goat antirat antibodies were not blocked with rat immune globulins, most Lin ${ }^{+}$cells appear Sca1 ${ }^{+}$ in Figure 1. This blocking step was omitted because only lineage-negative cells were isolated, and the blocking step did not affect the fluorescence or the number of the cells in the sort windows in preliminary experiments.

\section{Pre-colony-forming cell assay}

Lin-Sca1 ${ }^{++}$or $\mathrm{Lin}^{-} \mathrm{Scal}^{++} \mathrm{kit}^{+}$cells were cultured in triplicate at approximately 50 cells per well in flat-bottom, 96-well plates in serum-free media (StemPro34; Gibco BRL), $50 \mathrm{ng} / \mathrm{mL}$ flt3L, $50 \mathrm{ng} / \mathrm{mL} \mathrm{TPO}$, and $10 \%$ $\mathrm{BHK} / \mathrm{KL}$ supernatant (containing kit ligand). Because the BHK/KL supernatant contained $10 \%$ FCS, the actual serum concentration in these cultures was still $1 \%$. Three hours after plating, the exact number of cells per well was determined by visually counting the cells at $\times 40$ magnification. After 5 days of liquid culture at $37^{\circ} \mathrm{C}$ and $5 \% \mathrm{CO}_{2}$, the cells were counted, and 500 cells were plated in methylcellulose cultures containing IMDM, IL-3 (10\% of WEHI 3B supernatant), GM-CSF (10\% of BHK/HM-5 supernatant), KL (10\% of BHK/MKL supernatant), erythropoietin (2 U/mL), FCS (20\%), anti-TGF- $\beta(10 \mu \mathrm{g} / \mathrm{mL})$, and 2-mercaptoethanol $\left(10^{-6} \mathrm{M}\right)$. After 8 days of incubation at $37^{\circ} \mathrm{C}$ in a humidified incubator with $5 \% \mathrm{CO}_{2}$, the cultures were scored for colony formation. The same lot of fetal bovine serum was used in all the experiments.
A

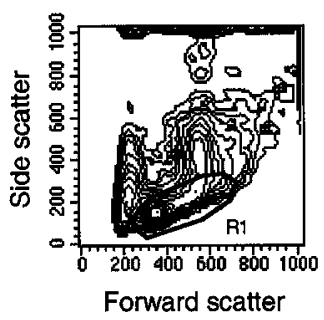

B

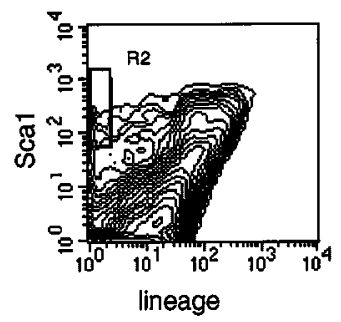

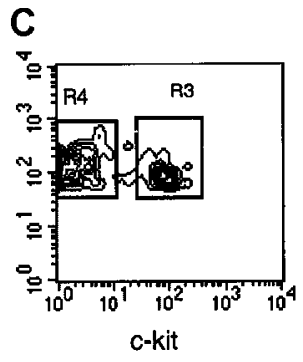

Figure 1. Sort windows used to isolate $\mathrm{Lin}^{-\mathrm{Sca} 1^{++}}$ $\mathrm{Lin}^{-} \mathrm{Scal}^{++} \mathbf{k i t}^{+}$, and $\mathrm{Lin}^{-} \mathrm{Scal}^{++}{ }^{+ \text {kit }^{-}}$cells. Cells with low to medium forward scatter (FSC) and low side scatter (SSC) (R1), negative green (lineage) fluorescence, orange (Sca1) fluorescence higher than twice the fluorescence level of cells stained with control antibodies (not shown) (R2), and positive red (c-kit) fluorescence (LSK+, R3) or negative red fluorescence ( $\mathrm{LSK}^{-}, \mathrm{R} 4$ ) were isolated. For the isolation of Lin-Sca1 ${ }^{++}$cells, cells falling into R1 and R2 were isolated. 


\section{Statistical analysis}

Student $t$ test for unpaired samples was used, unless indicated otherwise.

\section{Congenic mice}

Congenic mice were generated by marker-assisted back-crossing ${ }^{22,23}$ using B6 and BXD-31Ty as founders. These B6.DBA/2-(D2Mit133-D2Mit200) congenic animals have a segment of chromosome 2 between 66.7 and 102 $\mathrm{cm}$, derived from DBA/2 on a B6 background, and were obtained after 5 generations of male back-crossing and genotype-based selection with genetic markers spaced approximately $20 \mathrm{cM}$ apart. Analysis of polymorphic simple sequence repeat elements was made by polymerase chain reaction using primers obtained from Research Genetics (Huntsville, AL).

\section{Quantitative trait analysis using BXD RI strains}

Recombinant inbred strains were generated by repeated inbreeding of $\mathrm{F} 2$ mice derived from 2 parental inbred strains, C57BL/6 (B6) and DBA/2 in the case of BXD RI strains. The genome of RI strains is composed of a patchwork of homozygous chromosome segments derived from either progenitor strain, with each of the RI lines having a unique combination of patches from the progenitor. Genetic maps are available for each RI strain that allow the mapping of traits specified by loci at which the parental strains are polymorphic. ${ }^{24,25}$ Linkage analysis is performed by determining the relevant trait in each of the strains of a set of RI strains, which results in a strain distribution pattern (SDP). The data are then analyzed using Map Manager QTb29ppc software developed by Manly. ${ }^{26}$ This software statistically analyzes the linkage of a given trait with previously typed polymorphic loci in the RI strains, which are inherited from either parental strain. This allows the assignment of a trait to a corresponding map position and the calculation of statistical significance. ${ }^{27}$

Statistical analysis. Because of the limited number of RI strains available, the number of SDPs to be compared, and, consequently, the complexity of testing required to establish concordance, a close or identical match in SDPs may occur by chance. ${ }^{27,28}$ As with all statistical comparisons, it is necessary to make a calculation of the probability that the observed result was a false-positive. Therefore, the genome-wide probability of obtaining the observed linkages by random chance, corresponding to an error threshold of $P=.05$, is calculated using the robust nonparametric permutation method developed by Churchill and Doerge,27 which is implemented in the Map Manager QTb29ppc software developed by Manly et $\mathrm{al}^{26}$ The peak logarithm of the likelihood of odds ratio (the LOD score) of the correctly ordered data obtained in our study is compared with the peak LOD scores computed for 5000 random permutations of the same data. As an example of this kind of analysis, if the actual data gave an LOD score of 6 and only 1 in 1000 random permutations exceeded this value, the genome-wide probability of a false-positive would be approximately 0.001 . The $P=.5$ error threshold, a level considered suggestive of a QTL, corresponds to a LOD score that is exceeded by the highest LOD scores of half the permutations. ${ }^{27}$

Interval mapping. A subroutine of the Map Manager QTb29ppc software, using computationally efficient regression equations, is used for mapping the QTLs. The probability of linkage between our trait under study and previously mapped genotypes was estimated at 1-cM intervals along the entire genome, except for the $\mathrm{Y}$ chromosome. The statistical power of linkage of the phenotype to individual genotypes (point-wise linkage statistics) should attain values of at least 0.0001 to reach a level of genome-wide statistical significance. . $7,28^{2}$

Mapping databases. Mapping databases were downloaded from www.nervenet.org/papers/bxn.html. ${ }^{29}$

\section{Results}

Responsiveness to early-acting factors and number of phenotypically defined progenitor cells in B6 and DBA/2 mice

A pre-colony-forming cell (pre-CFC) assay was used to measure proliferation and differentiation capacity of $\mathrm{Lin}^{-} \mathrm{Sca}{ }^{++} \mathrm{kit}^{+}$ cells, ${ }^{16-18}$ isolated by flow cytometric cell sorting according to the sort windows shown in Figure 1, from $\mathrm{B} 6$ and DBA/2 mice. In this assay, Lin-Sca1 ${ }^{++}$kit $^{+}$cells were cultured in liquid cultures supported by the early-acting factors KL, flt3L, and TPO. ${ }^{19-21}$ After 5 days of culture, the cells were counted and plated in methylcellulose assays supported by KL, IL-3, GM-CSF, erythropoietin, and neutralizing anti-TGF- $\beta$ antibodies for the determination of $\mathrm{CFC}$ content. $\mathrm{B} 6$ and DBA/2 mice were chosen for these studies because of the known strain-dependent variation in the hematopoietic system between these 2 mouse strains ${ }^{2-6,8,9}$ and because of the availability of a relatively large set of BXD RI strains together with a dense map of polymorphic markers. ${ }^{24-26,29}$

Lin $^{-}$Sca1 ${ }^{++}$kit $^{+}$cells from B6 mice proliferated better in response to flt3L, KL, and TPO than $\mathrm{Lin}^{-} \mathrm{Sca1}{ }^{++} \mathrm{kit}^{+}$cells from $\mathrm{DBA} / 2$ mice $(P=.01, \mathrm{n}=12$, paired $t$ test; Figure $2 \mathrm{~A})$. The difference in CFC generation was not statistically significant ( $\mathrm{n}=12$, Figure 2A). Most colonies generated were myeloid, with $20 \%$ to $40 \%$ macroscopic colonies derived from high proliferative potential cells. During these experiments, we also recorded the number of sorted $\mathrm{Lin}^{-} \mathrm{Sca}{ }^{++} \mathrm{kit}^{+}$events, as determined on the counter on the cell sorter, as well as the frequency of Lin $^{-}$Sca1 ${ }^{++}$kit $^{+}$cells. The frequency (not shown) and the absolute number (Figure $2 \mathrm{~B}$ ) of $\mathrm{Lin}^{-} \mathrm{Sca}^{++}{ }^{+\mathrm{kit}^{+}}$cells were 2 -fold higher in B6 than in $\mathrm{DBA} / 2$ mice $(P=.04, \mathrm{n}=8)$. Long-term repopulating stem cells are enriched in the $\mathrm{Lin}^{-} \mathrm{Sca}^{++} \mathrm{kit}^{+}$fraction of bone marrow cells. ${ }^{16-18}$ In addition, a c-kit ${ }^{-}$subpopulation of Lin ${ }^{-}$Sca1 ${ }^{++}$ cells has been identified by Randall and Weissman that does not proliferate in vitro and that has no detectable long-term repopulating capacity. ${ }^{30}$ In addition, in our hands, these cells did not respond to flt3L, KL, or TPO at all ( $\mathrm{n}=16$, not shown). The biologic significance of this population is unknown. We noticed, however, that the number of $\mathrm{Lin}^{-} \mathrm{Scal}^{++} \mathrm{kit}^{-}$cells was also 2-fold higher in B6 than in DBA/2 mice $(P=.04, \mathrm{n}=8$, Figure $2 \mathrm{~B})$. When, in a separate set of experiments, the total number of $\mathrm{Lin}^{-} \mathrm{Sca} 1^{++}$cells was quantified, the number in B6 mice was again 2-fold higher than in DBA/2 mice $(P=.02, \mathrm{n}=6$, Figure $2 \mathrm{~B})$. These data suggest that the size of the $\mathrm{Lin}^{-} \mathrm{Sca} 1^{++}$population as a whole is genetically determined. An alternative explanation might be that B6 and DBA/2 mice simply differ in the level of expression of Sca1, so that

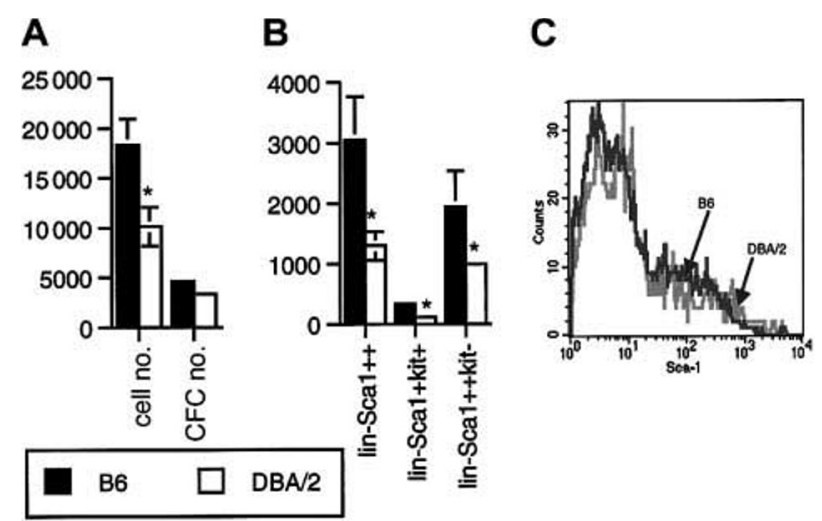

Figure 2. Proliferative capacity and number of $\mathrm{Lin}^{-} \mathrm{Scal}^{++} \mathrm{kit}^{+}$and Lin $^{-}$Scal $^{++}$kit $^{-}$cells in B6 and DBA/2 mice. (A) Number of cells and CFCs obtained after 5 days of liquid culture of $50 \mathrm{Lin}^{-} \mathrm{Scal}{ }^{++} \mathrm{kit}^{+}$cells from B6 and DBA/2 mice supported by $\mathrm{KL}$, flt3L, and TPO. Results are given as mean \pm SEM ( $n=12$ independent triplicate experiments; ${ }^{*}$ significantly different from $B 6$, paired $t$ test). (B) Absolute number of $\mathrm{Lin}^{-} \mathrm{Sca} 1^{++}, \mathrm{Lin}^{-} \mathrm{Scal}^{++} \mathrm{kit}^{+}$, and $\mathrm{Lin}^{-} \mathrm{Scal}^{++} \mathrm{kit}^{-}$cells in $\mathrm{B} 6$ and DBA/2 mice as determined by flow cytometric cell sorting (mean $\pm \mathrm{SEM}, \mathrm{n}=8$ independent experiments in which the bone marrow pooled from at least 2 mice was used; *significantly different from B6, paired $t$ test). (C) Range of expression levels of $\mathrm{Sca} 1$ on $\mathrm{Lin}^{-}$bone marrow cells from $\mathrm{B} 6$ and DBA/2 mice. 


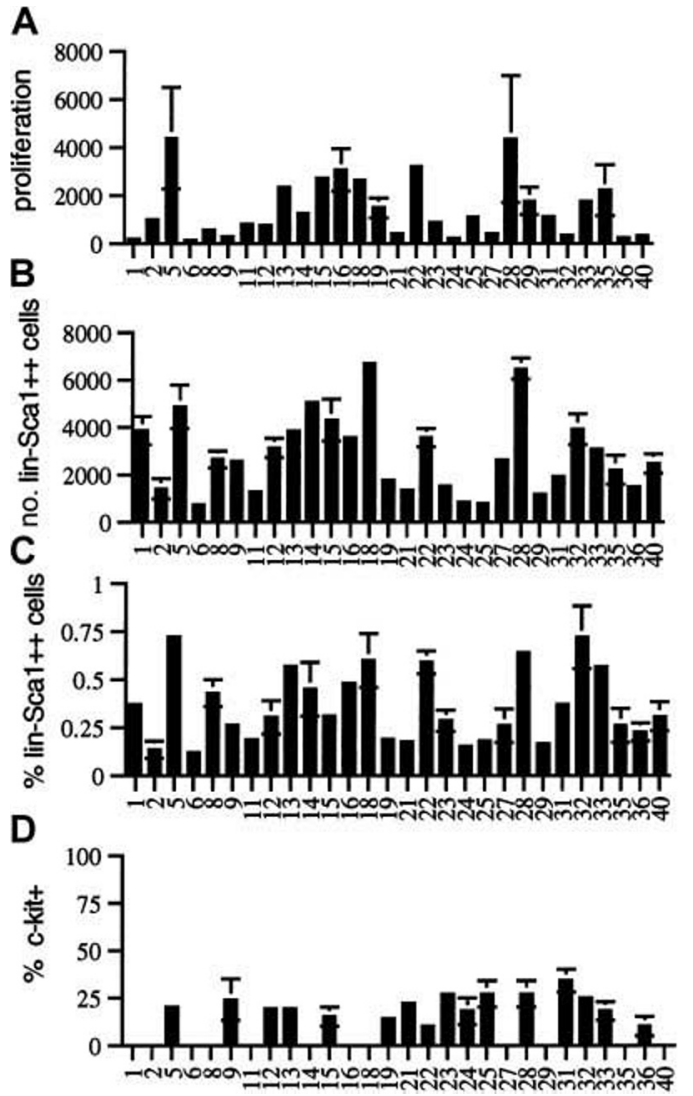

Figure 3. Strain-distribution patterns in BXD mice. Strain-distribution pattern of (A) the proliferation of $\mathrm{Lin}^{-} \mathrm{Sca} 1^{++}$cells in response to $\mathrm{KL}$, flt3L, and TPO (expressed as cell number after 5 days of culture per 50 input $\mathrm{Lin}^{-} \mathrm{Sca} 1^{++}$cells), (B) the absolute number of $\mathrm{Lin}^{-} \mathrm{Sca}{ }^{++}$cells, $(\mathrm{C})$ the relative fraction of $\mathrm{Lin}^{-} \mathrm{Sca}{ }^{++}$cells, and (D) the

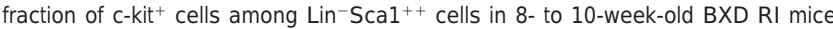
(mean \pm SEM, $\mathrm{n}=3$ independent experiments in which the bone marrow pooled from at least 2 mice was used).

it would appear that DBA/2 mice have a lower number of Sca1 ${ }^{++}$ cells. However, the range of expression levels of Sca1 on Lin ${ }^{-}$cells in $\mathrm{B} 6$ and DBA/2 mice (Figure $2 \mathrm{C}$ ) was identical. It is therefore unlikely that the observed 2-fold variation in the numbers of $\mathrm{Lin}^{-} \mathrm{Sca} 1^{++}$cells was caused by variation in the relative level of expression of the Sca1 antigen.

\section{Quantitative trait analysis in BXD RI strains}

To further investigate potential genetically determined variation in the cytokine responsiveness and number of $\mathrm{Lin}^{-} \mathrm{Sca} 1^{++}$cells, we performed quantitative trait analysis using BXD RI strains. Twentyeight BXD RI strains were analyzed for responsiveness to the early-acting factors flt3L, KL, and TPO, for the absolute number and frequency of $\mathrm{Lin}^{-} \mathrm{Sca}^{++}$cells, and for the fraction of c-kit ${ }^{+}$ cells among $\mathrm{Lin}^{-} \mathrm{Sca} 1^{++}$cells. In all RI strains, at least 3 independent experiments were performed in triplicate using bone marrow pooled from at least 2 individual mice in each experiment. The strain distribution patterns are shown in Figure 3. Because $\mathrm{Lin}^{-} \mathrm{Sca} 1^{++}$kit $^{-}$cells do not respond to early-acting cytokines at all $(\mathrm{n}=16)$, only $\mathrm{Lin}^{-} \mathrm{Sca}^{++}{ }^{+} \mathrm{kit}^{+}$cells are responsible for cell proliferation and CFC generation from $\mathrm{Lin}^{-} \mathrm{Sca}{ }^{++}$cells. Table 1 summarizes the map positions obtained.

There was wide phenotypic variation among BXD RI strains for proliferative capacity in response to flt3L, KL, and TPO. Cell number per 50 input $\mathrm{Lin}^{-} \mathrm{Sca} 1^{++}$cells after 5 days of culture ranged from $124 \pm 72$ (BXD6) to $4369 \pm 2119$ (BXD5) (Figure $3 \mathrm{~A})$. CFC output completely paralleled cellular proliferation, and no strain-dependent variation was noted in the secondary cloning efficiency (not shown). It was surprising that up to 35-fold variation in the proliferative capacity was seen among BXD RI strains (Figure $3 \mathrm{~A}$ ), whereas there was only a small difference in this trait among the 2 progenitor strains (Figure 2A), indicating that this is a multigenic, quantitative trait. For multigenic traits, phenotypic spread among BXD strains actually represents the phenotypic spread in the F2 generation, not of the progenitor strains. Therefore, extensive phenotypic variation can be seen within the RI strains, even when the phenotype of the 2 progenitor strains is not significantly different for that trait. The reason is that RI strains may have most of the positive or most of the negative alleles contributing to a given trait, whereas the 2 progenitor strains may have both positive and negative alleles for that trait. ${ }^{24,25} \mathrm{~A}$ major QTL for proliferative capacity was found on chromosome 2, with a likelihood ratio statistic (LRS) in the significant range as determined by permutation analysis. Interval mapping showed the highest LRS value (18.8) between D2Mit495 (73.2 cM) and D2Mit411 (77.6 cM) (Table 1). The donor of the high allele was B6.

Among the 28 BXD RI strains studied, the number of Lin $^{-}$Sca1 ${ }^{++}$cells varied from $721 \pm 86$ (BXD6) to $6472 \pm 447$ (BXD28) cells per femur (Figure 3B). The percentage of $\mathrm{Lin}^{-} \mathrm{Sca} 1^{++}$cells in the bone marrow (Figure $3 \mathrm{C}$ ) correlated well with the absolute number of $\mathrm{Lin}^{-} \mathrm{Sca}{ }^{++}$cells $(r=0.870, P<.0001$, not shown). This correlation indicates that the fraction of $\mathrm{Lin}^{-} \mathrm{Sca}^{++}$ cells is a good measure of the absolute number of $\mathrm{Lin}^{-} \mathrm{Sca} 1^{++}$cells and that our data cannot be explained by genetically determined variation in bone marrow cellularity. In addition, the range of expression of Sca1 was the same in all BXD strains, indicating again that differences in the expression levels of Scal cannot explain the variation in the number of $\mathrm{Lin}^{-} \mathrm{Scal}^{++}$cells. Although there was some variation in the ratio of c-kit ${ }^{+} / \mathrm{c}^{-\mathrm{kit}^{-}}$cells among

Table 1. Overview of mapped QTLs

\begin{tabular}{|c|c|c|c|c|c|c|c|}
\hline Trait & Chromosome & Markers & CM & $\begin{array}{l}\text { Maximum } \\
\text { LRS* }\end{array}$ & $P \dagger$ & High allele & Significance \\
\hline \multirow[t]{3}{*}{ No. Lin-Scal ${ }^{++}$cells } & 2 & D2Mit495-D2Mit411 & $73.2-77.2$ & 9.4 & .002 & B6 & Suggestive \\
\hline & 4 & D4Mit33-D4Mit343 & $77.5-79.0$ & 15.9 & .0001 & $\mathrm{DBA} / 2$ & Suggestive \\
\hline & 7 & D7Mit17-D7Mit238 & $51.0-53.0$ & 9.2 & .002 & B6 & Suggestive \\
\hline \multirow[t]{2}{*}{ flt3L/KL/TPO response } & 2 & D2Mit495-D2Mit411 & $73.2-77.2$ & 18.8 & .00007 & B6 & Significant \\
\hline & $x$ & ND & ND & ND & ND & DBA $/ 2 \ddagger$ & Significantł \\
\hline
\end{tabular}

ND indicates not determined.

*As determined by interval mapping.

$\dagger P$ value for point-wise linkage statistics.

‡Determined in reciprocal male $\mathrm{F} 1$ hybrids.

$\S$ According to published criteria. ${ }^{27,28}$ 
A

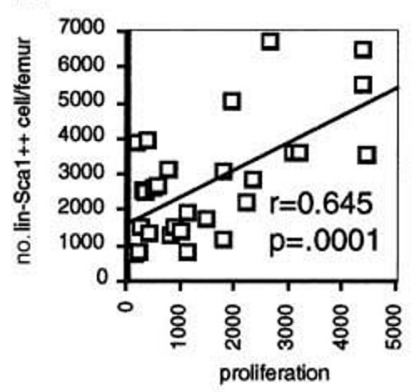

B

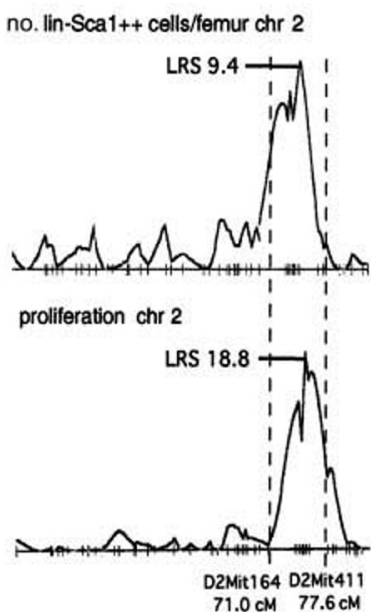

Figure 4. Responsiveness to early-acting factors and number of Lin-Sca1 ${ }^{++}$ cells in BXD RI strains. (A) Correlation between the responsiveness to flt3, KL, and TPO and number of $\mathrm{Lin}^{-} \mathrm{Sca}{ }^{++}$cells per femur among BXD RI strains. (B) LRS along chromosome 2 (chr 2) for the number of $\mathrm{Lin}^{-} \mathrm{Sca}{ }^{++}$cells per femur (top) and proliferation (bottom)

Lin $^{-}$Sca1 ${ }^{++}$cells in BXD RI strains, no statistically significant differences were found (Figure 3D; only strains in which 3 experiments were performed are shown). Variation in the fraction of c-kit-expressing $\mathrm{Lin}^{-} \mathrm{Sca} 1^{++}$cells thus cannot explain the 35 -fold variation in proliferative capacity of $\mathrm{Lin}^{-} \mathrm{Sca}{ }^{++}$cells. The 9 -fold variation in the number of Lin $^{-}$Sca1 ${ }^{++}$cells among BXD RI strains compared with the only 2-fold difference between the 2 progenitor strains, B6 and DBA/2, suggest that the number of Lin $^{-} \mathrm{Sca}^{++}$cells is a complex multigenic trait.

Quantitative trait analysis was performed for the number of Lin $^{-}$Sca $1^{++}$cells using the average values for the 3 independent experiments performed in each BXD strain (Table 1). This analysis revealed a highly suggestive QTL for the number of Lin ${ }^{-}$Sca1 ${ }^{++}$ cells on chromosome 4 (Table 1). The highest value of the LRS (15.9) by interval mapping was found between D4Mit33 (77.5cM) and D4Mit343 $(79 \mathrm{cM})$. This was just short of significant, for which an LRS of 16.6 was required according to permutation analysis. ${ }^{27}$ The frequency of $\mathrm{Lin}^{-} \mathrm{Sca} 1^{++}$cells mapped to the same region of chromosome 4 with a suggestive level of significance (LRS 12.7). The donor of the high allele was DBA/2. A second suggestive QTL (LRS 9.4 for absolute number and 11.4 for frequency of $\mathrm{Lin}^{-} \mathrm{Sca} 1^{++}$ cells) was found on chromosome 2, between D2Mit495 (73.2 cM) and D2Mit411 $(77.6 \mathrm{cM})$. The donor of the high allele at this locus was B6. A third, weaker QTL was found on chromosome 7 between D7Mit17 (51 cM) and D7Mit238 (53 cM); B6 was the donor of the high allele (LRS 9.2). The level of significance of the association with all 3 loci was in the suggestive range according to published criteria. This means that the association is likely but needs confirmation. ${ }^{25,27,28}$ However, the same 3 loci were also found, again with a level of statistical significance in the suggestive range, when each round of mapping was analyzed individually. Therefore, each of these QTLs has been confirmed in successive, independent rounds of linkage analysis. ${ }^{25}$

Interestingly, the QTL on chromosome 2 for the number of $\mathrm{Lin}^{-} \mathrm{Sca} 1^{++}$cells overlaps with the QTL on chromosome 2 for the proliferative capacity of $\mathrm{Lin}^{-} \mathrm{Sca} 1^{++}$cells. In addition, there was a significant correlation between proliferation and the number and frequency of $\mathrm{Lin}^{-} \mathrm{Sca} 1^{++}$cells among BXD RI strains (Figure 4A; data shown for absolute number of $\mathrm{Lin}^{-} \mathrm{Sca} 1^{++}$cells), and the pattern of LRS values along chromosome 2 was virtually identical for both traits (Figure 4B). Taken together, these data may suggest that genetic variation in the responsiveness to early-acting cytokines contributes to the regulation of the absolute number of Lin ${ }^{-}$Sca1 ${ }^{++}$cells among BXD RI strains.

\section{Confirmation of a locus on chromosome 2 determining the number of $\mathrm{Lin}^{-} \mathrm{Scal}^{++}$kit $^{+}$cells in congenic mice}

Congenic mice $22,23,25$ were constructed in the laboratory of GvZ, where a segment from chromosome 2 between D2Mit133 (66.7 $\mathrm{cM}$ ) and D2Mit200 (102 cM), encompassing the region containing a QTL for the number of $\mathrm{Lin}^{-} \mathrm{Sca} 1^{++}$cells and for their proliferative capacity (Table 1), from DBA/2 mice was crossed onto the B6 background (B6.DBA/2-[D2Mit133-D2Mit200] mice). We determined whether introgression of this DBA/2-derived segment of chromosome 2 onto the $\mathrm{B} 6$ background would change the number of $\mathrm{Lin}^{-} \mathrm{Sca} 1^{++}, \mathrm{Lin}^{-} \mathrm{Sca} 1^{++} \mathrm{kit}^{+}$, and $\mathrm{Lin}^{-} \mathrm{Sca}^{++}{ }^{+} \mathrm{kit}^{-}$cells and their responses to flt3L, KL, and TPO toward the values obtained for DBA/2 (Figure 2). Mice congenic for the distal region of chromosome 4 or chromosome 7 are not yet available.

As shown in Figure 5, congenic mice had significantly fewer Lin $^{-}$Sca ${ }^{++}{ }^{+}$it $^{+}$cells $(P=.0075)$ than B6 mice, whereas the difference in the number of Lin-Sca1 $^{++}$kit $^{-}$cells $(P=.1)$ and total Lin $^{-}$Sca1 ${ }^{++}$cells $(P=.07)$ was at the limit of statistical significance by paired $t$ test. Our data thus confirm that the segment of chromosome 2 between 66.8 and $107 \mathrm{cM}$ contains at least one gene that contributes to the regulation of the pool size of $\mathrm{Lin}^{-} \mathrm{Sca} 1^{++} \mathrm{kit}^{+}$cells and possibly also of $\mathrm{Lin}^{-} \mathrm{Sca}^{++} \mathrm{kit}^{-}$cells, that this gene shows allelic variation between $\mathrm{B} 6$ and $\mathrm{DBA} / 2$ mice, and that DBA/2 was the donor of the low allele for this trait.

We anticipated seeing lower proliferative capacity and CFC generation in $\mathrm{Lin}^{-} \mathrm{Sca}^{++} \mathrm{kit}^{+}$cells from congenic mice than from B6 mice. However, proliferation and CFC generation were identical in B6 and congenic mice (not shown, $\mathrm{n}=8$ ). The QTL on chromosome 2 for proliferative capacity could thus not be confirmed in congenic mice.

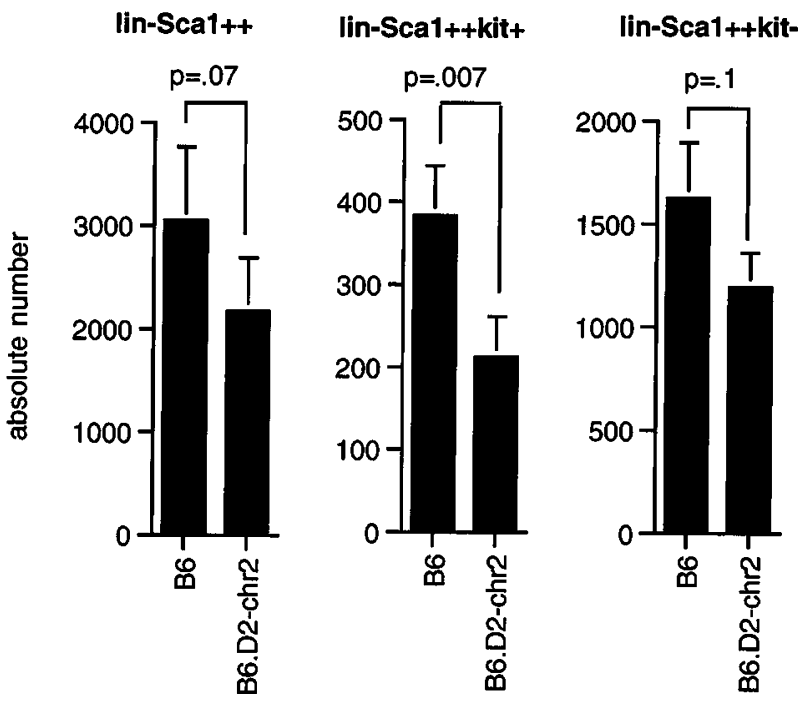

Figure 5. Number of $\mathrm{Lin}^{-} \mathrm{Sca}{ }^{++}, \mathrm{Lin}^{-} \mathrm{Scal}^{++} \mathrm{kit}^{+}$, and $\mathrm{Lin}^{-} \mathrm{Scal}^{++} \mathrm{kit}^{-}$cells in congenic mice. (A) Average number (mean $\pm \mathrm{SEM}, \mathrm{n}=8$ ) of $\mathrm{Lin}^{-} \mathrm{Sca}{ }^{++}$ $\mathrm{Lin}^{-} \mathrm{Scal}^{++} \mathrm{kit}^{+}$, and $\mathrm{Lin}^{-} \mathrm{Scal}{ }^{++} \mathrm{kit}^{-}$cells per femur in B6 and B6.DBA/2-(D2Mit133$\mathrm{D} 2 \mathrm{Mit200}$ ) congenic mice (the latter are indicated in the figure as B6.D2-chr2). Cell numbers were determined by cell sorting. $P$ values (paired $t$ test) are given at the tops of the figures. 
Evidence for $\mathrm{X}$-linked genetic factors in the regulation of the response of primitive hematopoietic stem and progenitor cells to early-acting cytokines in mice

Alleles of unknown X-linked genes confer a selective advantage to hematopoietic stem cells in vivo in humans and in cats. ${ }^{10-15}$ No models or in vitro assays are available to investigate X-linked regulation of hematopoiesis in the mouse. Furthermore, quantitative trait analysis with the available number of BXD RI strains does not detect all QTLs involved in complex traits. ${ }^{24,25}$ We therefore tried to establish a model to detect X-linked allelic variation in the hematopoietic stem cell compartment by investigating whether reciprocal male B6D2F1 and D2B6F1 mice differed in the number of $\mathrm{Lin}^{-} \mathrm{Sca}{ }^{++} \mathrm{kit}^{+}$and $\mathrm{Lin}^{-} \mathrm{Sca} 1^{++} \mathrm{kit}^{-}$cells and in the responsiveness of $\mathrm{Lin}^{-} \mathrm{Sca}^{++} \mathrm{kit}^{+}$to early-acting cytokines. Female D2B6F1 and B6D2F1 mice are heterozygous in all their loci, and $\mathrm{X}$ inactivation is random. ${ }^{31}$ Therefore, the phenotype of reciprocal female F1 hybrids will be the same, unless there is allelic variation in imprinted genes, so that a parent-of-origin effect is observed. Reciprocal male F1 hybrids, however, differ in the origin of the unique $\mathrm{X}$ chromosome (B6 in B6D2F1 mice, and $\mathrm{DBA} / 2$ in D2B6F1 mice). Phenotypic variation between reciprocal male F1 hybrids is thus most likely caused by allelic variation at X-linked loci. Theoretically, it is also possible that allelic variation at Y-linked loci exists.

The numbers of $\mathrm{Lin}^{-} \mathrm{Sca}{ }^{++}{ }^{+} \mathrm{kit}^{+}$and $\mathrm{Lin}^{-} \mathrm{Sca} 1^{++}{ }^{+} \mathrm{kit}^{-}$cells in female and male F1 hybrids were intermediate between those for the parental strains, indicating that it is unlikely that X-linked genes regulate the number of $\mathrm{Lin}^{-} \mathrm{Sca}^{++} \mathrm{kit}^{+}$or $\mathrm{Lin}^{-} \mathrm{Sca}{ }^{++} \mathrm{kit}^{-}$cells in vivo (not shown, $\mathrm{n}=8$ ). Proliferation (Figure $6 \mathrm{~A}$ ) and $\mathrm{CFC}$ production (Figure 6B) were similar in female B6D2F1 and D2B6F1 mice $(n=4)$. This is in accordance with complete heterozygosity in autosomal loci and random $\mathrm{X}$ inactivation in female F1 hybrids. ${ }^{31}$ In contrast to female F1 hybrid mice, however, proliferation (Figure 6A) and $\mathrm{CFC}$ generation (Figure 6B) in Lin $^{-}$Sca1 ${ }^{++}$kit $^{+}$cells from male D2B6F1 mice were significantly higher than in $\mathrm{Lin}^{-} \mathrm{Sca}^{++}{ }^{+} \mathrm{kit}^{+}$cells from male B6D2F1 mice $(\mathrm{n}=8)$. Because male D2B6F1 and B6D2F1 mice are heterozygous in all their autosomal loci but differ in the origin of the $\mathrm{X}$ chromosome (B6 in B6D2F1 mice and DBA/2 in D2B6F1 mice), these data suggest that at least one $\mathrm{X}$-linked locus contributes to the regulation of the response of $\mathrm{Lin}^{-} \mathrm{Sca} 1^{++} \mathrm{kit}^{+}$cells to early-acting factors and that the donor of the high allele is DBA/2. As mentioned before, genetic variation in Y-linked genes could theoretically also explain our data. Although this cannot be formally excluded, the involvement of Y-linked genes is less likely given the paucity of somatically active genes on the Y chromosome. $^{32}$ In addition, if allelic variation between $\mathrm{DBA} / 2$ and $\mathrm{B} 6$ mice on the $\mathrm{Y}$ chromosome plays a role, then one would expect a

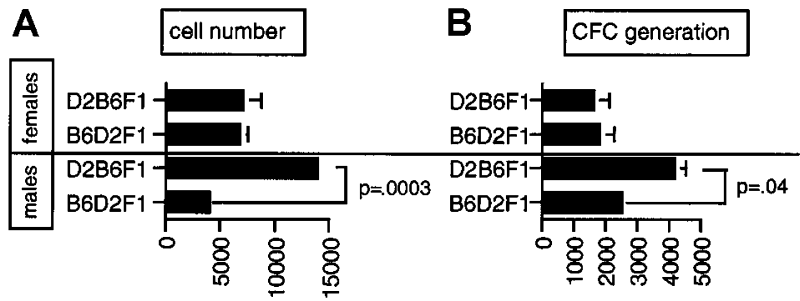

Figure 6. Difference in proliferative capacity in reciprocal male and female $F$ hybrid mice. Cell proliferation (A) and CFC generation (B) per 50 input $\mathrm{Lin}^{-} \mathrm{Scal}^{++} \mathrm{kit}^{+}$ cells isolated from male and female D2B6F1 and B6D2F1 hybrid mice. Results are given as mean \pm SEM. $P$ values indicated on the figure were obtained by paired $t$ test ( $\mathrm{n}=4$ for female D2B6F1 and B6D2F1 mice; $\mathrm{n}=8$ for male D2B6F1 and B6D2F1 mice) differential effect of sex on this trait in $\mathrm{B} 6$ and DBA/2 mice, which was not the case (not shown).

\section{Discussion}

In this study, we have identified 2 significant traits involving the hematopoietic stem and progenitor cell compartment that show quantitative variation: (1) responsiveness to early-acting hematopoietic cytokines and (2) absolute number of Lin $^{-}$Sca1 ${ }^{++}$cells (both c-kit ${ }^{+}$and c-kit ${ }^{-}$subpopulations).

Variation in the fraction of $\mathrm{Lin}^{-} \mathrm{Sca}^{+}$cells among inbred mouse strains was already noted previously by Spangrude and Brooks. ${ }^{33}$ Lin $^{-}$Sca1 ${ }^{++}$cells are highly enriched in stem and progenitor cells ${ }^{16-18}$ but are still heterogeneous. Long-term repopulating cells are thought to be $\mathrm{Lin}^{-} \mathrm{Sca} \mathrm{1}^{++} \mathrm{kit}^{\text {high }}, 1,16-18$ though some reports suggest that a $\mathrm{kit}^{-}$precursor of kithigh repopulating stem

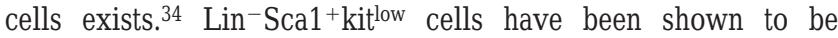
enriched in early-lymphoid precursors, ${ }^{35}$ though lymphoidcommitted precursors have recently been identified in the Lin ${ }^{-}$kithigh population as well. ${ }^{36}$ A Lin ${ }^{-}$Sca1 ${ }^{+}$kit $^{-}$population has been described by Randall and Weissman ${ }^{30}$ in steady state bone marrow. This mystery population is quiescent, increases in size with aging, does not proliferate in vitro, and does not contain long-term repopulating activity. We did indeed reproduce these findings (J.C.L. and H.-W.S., unpublished observations, December 2001). The functional significance of this $\mathrm{Lin}^{-} \mathrm{Sca}^{++}{ }^{+} \mathrm{kit}^{-}$population is unknown. We show here that at least 3 loci determine the total number of $\mathrm{Lin}^{-} \mathrm{Sca}{ }^{++}$cells: one on chromosome 2, one on chromosome 7, and one on chromosome 4. Because we found that there was little variation in the ratio of $\mathrm{kit}^{+} / \mathrm{kit}^{-}$cells among $\mathrm{Lin}^{-}$Sca1 ${ }^{++}$cells, despite a 10 -fold variation in the total number of Lin $^{-}$Sca1 ${ }^{++}$cells in BXD strains (Figure 3 ), we conclude that the number of $\mathrm{Lin}^{-} \mathrm{Sca} 1^{++} \mathrm{kit}^{+}$and $\mathrm{Lin}^{-} \mathrm{Sca}{ }^{++}{ }^{+} \mathrm{kit}^{-}$cells are regulated by at least partially overlapping genetic mechanisms. Indeed, the number of $\mathrm{Lin}^{-} \mathrm{Sca}^{++} \mathrm{kit}^{+}$and $\mathrm{Lin}^{-} \mathrm{Sca} 1^{++} \mathrm{kit}^{-}$cells is 2 -fold higher in B6 than in DBA/2 mice. These mechanisms are not necessarily identical, nor do they necessarily affect each subpopulation to the same extent. In congenic mice, we find a significant effect of the DBA-derived segment of chromosome 2 on the number of $\mathrm{Lin}^{-} \mathrm{Sca}{ }^{++}{ }^{+} \mathrm{kit}^{+}$cells, but the effect on the number of Lin $^{-}$Sca1 ${ }^{++}$kit $^{-}$and total Lin ${ }^{-}$Sca1 ${ }^{++}$cells numbers is at the limit of statistical significance, possibly suggesting that this locus mainly regulates the number of $\mathrm{Lin}^{-} \mathrm{Sca} 1^{++} \mathrm{kit}^{+}$cells. This will, of course, be reflected in the size of the total $\mathrm{Lin}^{-} \mathrm{Sca} 1^{++}$population, for which we have demonstrated linkage on chromosome 2 in BXD $\mathrm{RI}$ strains. Although the biologic significance of a small change in the number of $\mathrm{Lin}^{-} \mathrm{Sca} 1^{++}$cells caused by a single locus may be limited, the combined effect of many loci affecting pool size of Lin $^{-}$Sca1 ${ }^{++}$cells, as in those BXD mice with extreme values, is likely to be important. In addition, the importance of our data lies in the fact that we show that multiple loci affecting the number of $\mathrm{Lin}^{-}$Sca1 ${ }^{++}$cells exist. This may lead to identification of the mechanisms that regulate the number of $\mathrm{Lin}^{-} \mathrm{Sca}{ }^{++}$cells and of the kit $^{+}$and kit $^{-}$subpopulations thereof.

The correlation between the response to flt3L, KL, and TPO and the number of $\mathrm{Lin}^{-} \mathrm{Sca}{ }^{++}$cells among BXD RI strains and the overlapping QTLs for both traits raise the possibility that the responsiveness to early-acting cytokines plays a role in determining the number of $\mathrm{Lin}^{-} \mathrm{Sca} 1^{++}$cells. However, a phenotype could be reproduced in mice congenic for the locus on chromosome 2 for the number of $\mathrm{Lin}^{-} \mathrm{Sca} 1^{++} \mathrm{kit}^{+}$and $\mathrm{Lin}^{-} \mathrm{Sca} 1^{++} \mathrm{kit}^{-}$cells, but not 
for proliferative capacity in response to $\mathrm{KL}$, flt3L, and TPO. The most likely explanation for the lack of a proliferative phenotype in the congenic mice is epistasis with other loci that show allelic variation. ${ }^{25,37}$ In addition, a locus was found on the $\mathrm{X}$ chromosome that clearly determines the response of $\mathrm{Lin}^{-} \mathrm{Sca} 1^{++} \mathrm{kit}^{+}$cells to early-acting factors, but it has no effect on the number of $\mathrm{Lin}^{-} \mathrm{Sca} 1^{++} \mathrm{kit}^{+}$or $\mathrm{Lin}^{-} \mathrm{Sca} 1^{++} \mathrm{kit}^{-}$cells. Our data thus suggest that genetic variation in the response to early-acting cytokines does

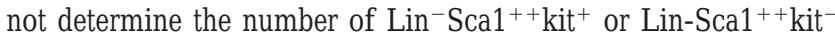
cells. This is in accordance with the data of Miller et $\mathrm{al}^{, 38}$ who showed that the number of stem cells, as defined by the $\mathrm{Lin}^{-} \mathrm{Sca}{ }^{+}{ }^{+} \mathrm{WGA}^{+}$phenotype, is similar in W mutant and wild-type mice. The correlation between the response to early-acting factors and the number of $\mathrm{Lin}^{-} \mathrm{Sca}{ }^{++}$cells is most likely explained by the fact that linked, but distinct, QTLs on chromosome 2 are major determinants for both traits.

Other QTLs related to hematopoiesis have been mapped to this segment of chromosome 2. In several studies, 8,39 a suggestive QTL on chromosome 2 at approximately $50 \mathrm{cM}$, regulating the increase of the number of CAFCd35 with aging in $\mathrm{B} 6$ and DBA/2 mice has been reported. Hasegawa et $\mathrm{al}^{9}$ identified a significant QTL that controls the efficiency of mobilization of hematopoietic progenitor cells in response to GM-CSF on chromosome 2 between 46 and 86 cM. ${ }^{9}$ It was recently shown that the mobilization of hematopoietic stem cells occurs after the M-phase of the cell cycle. ${ }^{40}$ Therefore, it is possible that an allele responsible for a higher responsiveness to early-acting factors also causes more efficient mobilization of hematopoietic stem cells. Potential candidate genes in this region of chromosome 2 known to play a role in hematopoiesis include jag1 (encoding the Notch ligand Jagged-1), ${ }^{41}$ bmp2 (encoding BMP-2), ${ }^{42}$ and the il1 complex (encoding IL- $1 \alpha$ and IL-1 $\beta$, respectively)..$^{43}$

QTL analysis, as reported here and by others, ${ }^{2-6,8,9}$ demonstrates differential genetic regulation of the size of the stem and progenitor cell compartment, as defined by immunophenotype on one hand and by functional assays on the other hand. Our data show that $\mathrm{Lin}^{-} \mathrm{Sca}^{++} \mathrm{kit}^{+}$cells are more numerous in $\mathrm{B} 6$ than in DBA/2 mice, confirming an earlier report in which the $\mathrm{WGA}^{++} \mathrm{Lin}^{-}$rho123low phenotype was used to isolate and identify the most primitive hematopoietic precursors. ${ }^{6}$ Yet, Lin $^{-}$Sca ${ }^{++}{ }^{+}$kit $^{+}$cells from DBA/2 mice repopulate syngeneic recipients more efficiently than those of B6 mice and generate more CAFCd35. ${ }^{44}$ In addition, DBA/2 mice show faster recovery after the administration of 5-fluorouracil than B6 mice ${ }^{44}$ and contain more CAFCd35 and LTC-IC than B6 mice. ${ }^{2,3,8}$ Conversely, data in allophenic mice suggest that on aging, B6-derived stem cells are more efficient at sustaining hematopoiesis. ${ }^{4,5}$ In CXB mice, long-term repopulation potential does not correlate with LTC-IC and CAFC numbers either. ${ }^{7}$ QTL analysis and subsequent identification of genes responsible for quantitative variation in the stem cell compartment, as defined by function and by phenotype, may provide novel insight into the regulation of hematopoiesis by helping to resolve these discrepancies. A possible explanation may be that the number of $\mathrm{Lin}^{-} \mathrm{Sca} 1^{++} \mathrm{kit}^{+}$cells is a reflection of stem and progenitor cell pool size in steady state conditions, whereas assays such as CAFC, LTC-IC, and long-term repopulation assay ${ }^{45}$ reflect, among other things, the capacity of the stem cell compartment to respond to hematopoietic stress.

The answer to whether the putative X-linked locus we identified here is the same as the locus that causes skewed $\mathrm{X}$ inactivation on aging in cats and in humans will have to await gene identification of the loci involved. However, given the importance of early-acting cytokines such as KL, flt3L, and TPO in vivo, ${ }^{19-21}$ allelic variation in one or more $\mathrm{X}$-linked genes affecting the responsiveness to these cytokines is an attractive candidate mechanism for the large genetic component in the skewed $\mathrm{X}$ inactivation in elderly females in humans ${ }^{11,15}$ and in cats. ${ }^{12}$ Our study suggests that an easy assaymeasurement of the responsiveness to early-acting cytokines in vitro-may allow mapping and identification of X-linked loci involved in the regulation of hematopoiesis.

In summary, we demonstrated genetic variation in the response to cytokines critical for hematopoiesis in vivo and in the pool size of cells belonging to a phenotype used to isolate essentially pure primitive progenitor and stem cells, and we identified loci that may be relevant to the regulation of hematopoiesis in steady state.

\section{References}

1. Morrison SJ, Uchida N, Weissman IL. The biology of hematopoietic stem cells. Ann Rev Cell Dev Biol. 1995;11:35-71.

2. de Haan G, Van Zant G. Intrinsic and extrinsic control of hematopoietic stem cell numbers: mapping of a stem cell gene. J Exp Med. 1997;186: 529-536.

3. Muller-Sieberg CE, Riblet R. Genetic control of the frequency of hematopoietic stem cells in mice: mapping of a candidate locus to chromosome 1.J Exp Med. 1996;171:1141-1150.

4. Van Zant G, Eldridge PW, Behringer RR, Dewey MJ . Genetic control of hematopoietic kinetics revealed by analysis of allophenic mice and stem cell suicide. Cell. 1983;35:639-645.

5. Van Zant G, Holland BP, Eldridge PW, Chen J). Genotype-restricted growth and aging patterns in hematopoietic stem cell populations of allophenic mice. J Exp Med. 1990;171:15471565.

6. Phillips R, Reinhart A, Van Zant G. Genetic control of murine hematopoietic stem cell pool sizes and cycling kinetics. Proc Natl Acad Sci U S A. 1992;89:11607-11611.

7. Chen J, Astle CM, Muller-Sieburg CE, Harrison $D E$. Primitive hematopoietic stem cell function in vivo is uniquely high in the $C X B-12$ mouse strain Blood. 2000;96:4124-4131.

8. Geiger H, True J, de Haan G, Van Zant G. Age- and stage-specific regulation patterns in hematopoietic stem cell hierarchy. Blood. 2001;98:29662972.

9. Hasegawa M, Baldwin TM, Metcalf D, Foote SJ . Progenitor cell mobilization by granulocyte colony-stimulating factor controlled by loci on chromosomes 2 and 11. Blood. 2000;95:18721874.

10. Champion KM, Gilbert J G, Asimakopoulos FA, Hinshelwood S, Green AR. Clonal haemopoiesis in normal elderly women: implications for the myeloproliferative disorders and myelodysplastic syndromes. Br J Haematol. 1997;97:920-926.

11. Christensen $\mathrm{K}$, Kristiansen $\mathrm{M}$, Hagen-Larsen $\mathrm{H}$, et al. X-linked genetic factors regulate hematopoietic stem-cell kinetics in females. Blood. 2000;95: 2449-2451.

12. Abkowitz J L, Taboada M, Shelton GH, Catlin SN Guttorp P, Kiklevich J V. An X chromosome gene regulates hematopoietic stem cell kinetics. Proc Natl Acad Sci U S A. 1998;95:3862-3866.

13. Abkowitz J L, Ott RM, Holly RD, Adamson J W. Clonal evolution following chemotherapy-induced stem cell depletion in cats heterozygous for glucose-6-phosphate dehydrogenase. Blood. 1988; 71:1687-1692.

14. Abkowitz J L, Linenberger ML, Persik M, Newton MA, Guttorp P. Behavior of feline hematopoietic stem cells years after busulfan exposure. Blood. 1993;82:2096-2103
15. Vickers MA, McLeod E, Spector TD, Wilson IJ Assessment of mechanism of acquired skewed $X$ inactivation by analysis of twins. Blood. 2001;97: 1274-1281.

16. Spangrude G] , Heimfeld S, Weissman IL. Purification and characterization of mouse hematopoietic stem cells. Science. 1988;241:58-62.

17. Trevisan M, Iscove NN. Phenotypic analysis of murine long-term hemopoietic reconstituting cells quantitated competitively in vivo and comparison with more advanced colony-forming progeny. J Exp Med. 1995;181:93-103.

18. Okada S, Nakauchi H, Nagayoshi K, et al. Enrichment and characterization of murine hematopoietic stem cells that express c-kit molecule. Blood. 1991;78:1706-1712.

19. Kimura S, Roberts AW, Metcalf D, Alexander WS. Hematopoietic stem cell deficiencies in mice lacking $\mathrm{c}-\mathrm{Mpl}$, the receptor for thrombopoietin. Proc Natl Acad Sci U S A. 1998;95:1195-1200.

20. Solar GP, Kerr WG, Zeigler FC, et al. Role of C$\mathrm{mpl}$ in early hematopoiesis. Blood. 1998;92:4-10.

21. Lyman SD, J acobsen SE. c-Kit ligand and Flt3 ligand: stem/progenitor cell factors with overlapping yet distinct activities. Blood.1998;91:1101-1134.

22. Markel P, Shu P, Ebeling C, et al. Theoretical and empirical issues for marker-assisted breeding of congenic mouse strains. Nat Genet. 1997;17:280-284.

23. Wakeland E, Morel L, Achey K, Yui M, Longmate J . 
Speed congenics: a classic technique in the fast lane (relatively speaking). Immunol Today. 1997; 18:472-477.

24. Silver LM. Mouse Genetics: Concepts and Applications. Oxford, England: Oxford University Press; 1995.

25. Moore KJ, Nagle DL. Complex trait analysis in the mouse: the strengths, the limitations and the promises yet to come. Annu Rev Genet. 2000;34: 653-686.

26. Manly KF, Olson J M. Overview of QTL mapping software and introduction to map manager QT. Mamm Genome. 1999;10:327-334.

27. Churchill GA, Doerge RW. Empirical threshold values for quantitative trait mapping. Genetics. 1994;138:963-971

28. Lander E, Kruglyak L. Genetic dissection of complex traits: guidelines for interpreting and reporting linkage results. Nat Genet. 1995;11:241-247.

29. Williams RW, Gu J, Qi S, Lu L. The genetic struc ture of recombinant inbred mice: high-resolution consensus maps for complex trait analysis. Release 1, J anuary 15, 2001 at www.nervenet.org/ papers/bxn.html or mickey.utmem.edu/papers/ bxn.html.

30. Randall TD, Weissman IL. Characterization of a population of cells in the bone marrow that phenotypically mimics hematopoietic stem cells: resting stem cells or mystery population? Stem Cells. 1998;16:38-48.

31. Avner P, Heard E. X-chromosome inactivation: counting, choice and initiation. Nat Rev Genet. 2001;1:59-67.

32. Charlesworth B, Charlesworth D. The degeneration of $Y$ chromosomes. Philos Trans R Soc Lond B Biol Sci. 2000;355:1563-1572.

33. Spangrude GJ, Brooks DM. Mouse strain variability in the expression of the hematopoietic stem cell antigen Ly6A/E by bone marrow cells. Blood. 1993;82:3327-3332.

34. Ortiz M, Wine JW, Lohrey N, Ruscetti FW, Spence ER, Keller J R. Functional characterization of a novel hematopoietic stem cell and its place in the c-Kit maturation pathway in bone marrow cell development. Immunity. 1999;10: 173-182.

35. Mojica MP, Perry SS, Searle AE, et al. Phenotypic distinction and functional characterization of pro-B cells in adult mouse bone marrow. J Immunol. 2001;166:3042-3051.

36. Medina KL, Garrett KP, Thompson LF, Rossi MI, Payne KJ , Kincade PW. Identification of very early lymphoid precursors in bone marrow and their regulation by estrogen. Nat Immunol. 2001; 2:718-724.

37. Flint J, Mott R. Finding the molecular basis of quantitative traits: successes and pitfalls. Nat Rev Genet. 2001;2:437-444.

38. Miller CL, Rebel VI, Lemieux ME, Helgason CD, Lansdorp PM, Eaves CI. Studies of W mutant mice provide evidence for alternate mechanisms capable of activating hematopoietic stem cells. Exp Hematol. 1996;24:185-194.
39. de Haan G, Van Zant G. Dynamic changes in mouse hematopoietic stem cell numbers during aging. Blood. 1999;93:3294-3301.

40. Wright DE, Cheshier SH, Wagers AJ, Randall TD, Christensen J L, Weissman IL. Cyclophosphamide/granulocyte colony-stimulating factor causes selective mobilization of bone marrow hematopoietic stem cells into the blood after $\mathrm{M}$ phase of the cell cycle. Blood. 2001;97:22782285.

41. Varnum-Finney $B$, Purton LE, Yu M et al. The Notch ligand, J agged-1, influences the development of primitive hematopoietic precursor cells. Blood. 1998;91:4084-4091.

42. Bhatia M, Bonnet $D, W u D$, et al. Bone morphogenetic proteins regulate the developmental program of human hematopoietic stem cells. J Exp Med. 1999;89:1139-1148.

43. Ruscetti FW, Dubois CM, J acobsen SE, Keller J R. Transforming growth factor beta and interleukin-1: a paradigm for opposing regulation of haemopoiesis. Baillieres Clin Haematol. 1992;5:702703.

44. de Haan G, Szilvassy SJ , Meyerrose TE, Dontje B, Grime B, Van Zant G. Distinct functional properties of highly purified hematopoietic stem cells from mouse strains differing in stem cell numbers. Blood. 2000;96:1374-1379.

45. Harrison DE. Evaluating functional abilities of primitive hematopoietic stem cell populations. Curr Top Microbiol Immunol. 1992;177:13-30. 